\title{
Rhétorique du monde muet dans « Le parti pris des choses » de Francis PONGE

\author{
Dr. Tarnime Mohamed Réda Taher
}

Département de langue française

Faculté de Pédagogie, Université d'Alexandrie

\section{Résumé}

Désigné par le «poète des choses », Francis PONGE tente de rendre compte de la beauté singulière qui émane des objets banals et quotidiens. Cette primauté reflète un double dessein : d'une part la volonté d'arracher l'homme à sa « vision anthropocentrique », d'autre part, l'engagement dans une tentative permettant de donner une voix à des éléments qui en sont privés grâce aux ressources cachées de la langue. Au-delà de la primauté apparente de l'objet se dissimule la prééminence du langage poétique. L'objectif de notre étude est de démontrer que l'ambition de l'auteur n'étant donc pas de reproduire des éléments quotidiens mais de fabriquer un nouvel objet poétique en manipulant le texte dans sa matérialité. Notre étude des principes de cette entreprise pongienne a adopté deux types d'approches sémiotique et stylistique que nous estimons se compléter et s'éclairer mutuellement. Cet angle de vue précis nous a permis d'aboutir à une meilleure explication des «qualités différentielles du texte pongien ».

Mots clés : Francis Ponge ; Prose ; XXème siècle ; titrologie ; stylistique ; rhétorique.

«Un texte est en effet la trace d'un discours où la parole est mise en scène $»^{1}$.

Tout au long de sa carrière d'écrivain, Francis PONGE a suivi un itinéraire particulièrement indépendant qui lui vaut une place de choix dans les débats philosophiques et littéraires. Sa démarche créatrice trouve ses racines dans son plus jeune âge. Dès 17 ans, il souffre d'une crise du mutisme et par la suite il affronte tant de difficultés à s'exprimer par la parole. Par conséquent, il échoue dans les épreuves qui exigent une communication verbale ${ }^{2}$. Quoique

1 MAINGUENEAU, Dominique, Le discours littéraire : Parotopie et scène d'énonciation, Armand Colin, 2004, p. 191. Sauf indication contraire, la ville d'édition dans notre étude est Paris.

2

Après avoir présenté une dissertation ayant pour titre L'art de penser par soi-même pour laquelle il a obtenu la meilleure note, ses études supérieures se sont terminées par 
complètement muet à l'oral, il est un brillant élève passionné par le Littré ${ }^{3}$ et les cours de rhétorique : "Renouer avec la parole prend alors la forme d'une résistance, et devient un exercice de rééducation verbale $»^{4}$. Dès lors, il éprouve un désir de s'exprimer tout en trouvant des sujets objectifs qui ne soient pas centrés sur lui-même. C'est pour cette raison qu'il décide d'être le porte-parole du monde muet et tente de rendre compte de la beauté singulière qui émane des objets banals et quotidiens.

Désigné par le «poète des choses $»^{5}$, PONGE manifeste un souci constant de retranscrire les qualités authentiques des réalités concrètes ou abstraites $^{6}$ afin de les « connaître objectivement $»^{7}$. Cette primauté reflète un double dessein : d'une part la volonté d'arracher l'homme à sa «vision

un double échec : il n'est pas parvenu à achever sa licence en philosophie et il a échoué au concours d'inscription à l'École normale supérieure en 1919. À ceci s'ajoute la crise affrontée à la suite de la mort de son père en 1923 pendant laquelle sa capacité langagière s'est profondément altérée, jusqu'à détruire complètement son rapport au langage et le rendre incapable de communiquer. Il a qualifié cette expérience amère par «La rage de l'expression », titre de l'un de ses ouvrages publié en 1952. Ainsi, « l'aphasie est à l'origine de son cuvre. » FARASSE, Gérard, VECK, Bernard, Guide d'un petit voyage dans l'œuvre de Francis Ponge, Presses universitaires du Septentrion, Collection Savoirs Mieux, Villeneuve d'Ascq, 1999, p. 71, une version électronique de l'ouvrage est disponible à travers le lien https://books.openedition.org/septentrion/51106, consulté le 3 mai 2020.

${ }^{3}$ Le Littré est un dictionnaire historique, étymologique et grammatical de la langue française élaboré par Emile Littré et datant de la fin du XIXème siècle. Il se démarque des dictionnaires purement explicatifs par le fait qu'il soit fortement imprégné par des explications et des citations littéraires très riches. Il est publié en 1871 en quatre volumes aux éditions Hachette. PONGE raconte : "Mon père avait, dans sa bibliothèque, le Littré, qui a eu une si grande importance pour moi, où j'ai trouvé un autre monde, celui des vocables, des mots, des mots français bien sûr, un monde aussi réel pour moi, aussi faisant partie du monde extérieur, du monde sensible. »Cf., respectivement, dictionnaire Hachette encyclopédique, 2001, p.p. 1099,1100, SOLLERS, Phillipe, Entretiens de Francis Ponge avec Philippe Sollers, Gallimard, Seuil, 1970, p. 46.

${ }^{4}$ RIVA, Magali, Francis Ponge : la méthode poétique, in La poésie scientifique de la gloire au déclin, Epistémocritique, littérature et savoirs, 2014, p. 448.

${ }^{5}$ COLLET, Michel, Francis Ponge : entre mots et choses, Champ Vallon, 1993, p. 7.

${ }^{6}$ Dans la poétique pongienne, le terme « chose » peut signifier des objets mais aussi des phénomènes naturels comme nous éclairerons plus loin. $C f$., infra, p. 8 .

7 CHARRON, Philippe, Représentation de l'objet chez Ponge : une pratique transparente du sens spécifique, Montréal, 2007, p. 4. 
anthropocentrique $\gg^{8}$ ce qui lui permet de se distancier de la forme de poésie lyrique ${ }^{9}$. D'autre part, l'écrivain s'engage dans une certaine tentative de donner une voix à des objets qui en sont privés à travers une sorte de "poésie impersonnelle $»^{10}$. Dans Le parti pris des choses ${ }^{11}$ Francis PONGE présente un assemblage de poèmes en prose ${ }^{12}$ publié en 1942, mais qui réunit des textes rédigés bien avant cette date ${ }^{13}$. Dans cet ouvrage, la préoccupation principale de l'auteur est de tenter de capter et de transposer la matérialité énigmatique des « choses » familières grâce aux ressources cachées de la langue. Il s'agit donc d'un certain « débat entre mots et choses $»^{14}$. La visée n'est pas de présenter les objets ordinaires mais de les «re/présenter », dans le double sens du terme : représentation comme incarnation et description mais également déclaration d'une nouvelle identité voire suggestion d'un regard original et d'une conception

${ }^{8}$ RIVA, Magali, art. cit., p. 442.

${ }^{9}$ Issue du romantisme, la poésie lyrique se fonde principalement sur les émotions personnelles de l'auteur. Selon PONGE, elle est complètement subjective et emploie un langage artificiel. Pour maintenir cette distance anti-lyrique, il refuse d'être qualifié comme « poète », et préfère le terme « savant ». Selon ses propres termes, il affirme : «Oui, je me veux moins poète que savant. Je désire moins aboutir à un poème qu'à une formule, qu'à un éclaircissement d'impressions. S'il est possible de fonder une science dont la matière serait les impressions esthétiques, je préfère être l'homme de cette science. » Dans notre analyse nous nous soumettrons à la volonté de PONGE et nous éviterons de le désigner par le terme poète, nous le substituerons par l'auteur ou l'écrivain. PONGE, Francis, La rage de l'expression (La Mounine), Gallimard, 1952, p. 425.

${ }^{10}$ RIVA, Magali, art. cit., p. 442.

11 PONGE, Francis, Le parti pris des choses, Gallimard, 1967, 170 pages. Les références aux poèmes du corpus seront marquées par le titre du texte suivi de la page, placés entre parenthèses.

${ }^{12}$ Le genre de la poésie en prose remonte au début du XIXème siècle, il découle d'une certaine volonté d'éviter l'emploi traditionnel de la langue et de profiter d'une liberté d'expression sans contraintes formelles telles la versification et les rimes.

13 Les poèmes réunis dans ce recueil sont rédigés entre 1924 et 1939 pendant la période de l'entre-deux guerres. Durant cette phase, PONGE se rapproche du surréalisme mais ne s'y souscrit pas. S'il partage avec ses adhérents quelques penchants dont le sentiment de révolte face aux évènements politiques et sociaux ainsi qu'une insistante volonté de renouvellement des moyens d'expression, il s'en distingue sur plusieurs plans. Alors que les surréalistes préfèrent les associations spontanées et donnent libre cours à l'émanation de l'inconscient et à l'écriture automatique, l'auteur manifeste un ardent besoin de rétablir une conception plus classique de la poésie où il exploite l'étymologie grecque et latine et puise ses images dans la mythologie.

${ }^{14}$ COLLET, Michel, op. cit., p. 9. 
novatrice de ces éléments. Pour ce faire, l'écrivain recourt aux caractéristiques physiques et linguistiques afin de nous faire savourer la beauté de ces objets :

«Le monde muet fait signe, il est scandaleusement négligé par tous les discours, la vie quotidienne du moindre objet ou animal est une source de connaissances inédites. (...) Il suffit d'écouter [la nature], de la regarder mieux, de s'apprendre soimême à son contact intime $»^{15}$.

L'intérêt que nous avons porté à ce corpus revient au fait qu'il retranscrit d'une manière littéraire un projet à la fois scientifique et linguistique puisque l'auteur affirme vouloir construire des « descriptions-définitions-objets-d'artlittéraire $»^{16}$. C'est en ce sens que nous avons voulu entreprendre la tâche de déceler les fondements de cette entreprise. L'itinéraire proposé dans cet article invite le lecteur à démarrer par une typologie des « choses » regroupées dans le recueil en nous basant sur une réflexion approfondie concernant l'un des lieux paratextuels les plus importants à savoir le titre. Ainsi, s'amorcera l'exploitation de cette "frange du texte $»^{17}$, qui, s'étendra graduellement et s'ouvrira pour s'élargir et englober l'étude des intertitres. Cette dernière nous permettra de catégoriser les éléments essentiels qui constituent les objets/sujets. Par la suite, nous opterons pour l'analyse des techniques de présentation et nous étudierons les procédés rhétoriques exhibés par l'auteur dans le but de distinguer clairement les figures de style dont il use plus fréquemment afin de transmettre la parole des objets ${ }^{18}$. Notre objectif vise à éviter tout risque de redite et de redondance ;

${ }^{15}$ SOLLERS, Philippe, "Ponge en abîme », Entretien à l'occasion de la publication des cuvres de Francis Ponge dans la Pléiade en 1999, version électronique disponible à travers le lien http://www.pileface.com/sollers/spip.php?article432, consulté le 3 mai 2020. Soulignons que les articles numériques, à travers les liens précisés, s'étalent sur une seule page web ; voilà pourquoi, dans les références aux articles en ligne, nous ne pourrons pas marquer la pagination.

16 PONGE, Francis, "My Creative Method ", in Euvres complètes, tome I, Gallimard, Bibliothèque de la Pléiade, 1999, p. 521. Il désirait «boucler une nouvelle Encyclopédie où science et poésie seraient réconciliées ». SOLLERS, Philippe, « Ponge en abîme », art. cit.

${ }^{17}$ Cette expression est empruntée à GENETTE qui s'inspire à son tour de Philippe LEJEUNE dans son ouvrage Le pacte autobiographique, Seuils, 1975, p. 45.

${ }^{18}$ Selon PONGE, le rôle primordial des poètes c'est d'être «les ambassadeurs du monde muet. »Cf., ROMESTAING, Alain, L'objet chez Francis Ponge: du «Parti- 
ainsi, nous nous limiterons à l'étude de quelques textes majeurs qui nous paraissent parmi les plus emblématiques de la logique scripturale pongienne.

\section{Du titre au texte :}

«Plus que d'une limite ou d'une frontière, il s'agit ici d'un seuil (...) qui offre à tout un chacun la possibilité d'entrer ou de rebrousser chemin ${ }^{19}$.

Si le paratexte constitue la porte d'entrée de l'univers exceptionnel du recueil, le titre serait le premier élément auquel est confronté le lecteur car il présente une idée intégrante et indissociable du sujet de l'ouvrage. L'étude de la titrologie ${ }^{20}$ donne une impression globale de l'œuvre littéraire avant la lecture. En effet, les théoriciens distinguent trois rôles essentiels de l'appareil titulaire : la fonction apéritive, abréviatrice et distinctive ${ }^{21}$.

De prime abord, Le parti pris des choses répond parfaitement aux trois fonctions précitées. Il annonce l'idée matrice du recueil sans trop dévoiler piquant ainsi la curiosité du lecteur, il résume les mots-clés à savoir parti pris et choses et enfin il singularise cette œuvre et la distingue. Le titre choisi pour coiffer l'ouvrage nous a incité à effectuer de nombreuses investigations, mais avant d'entamer l'analyse de l'appareil titulaire, il importe de souligner un aspect particulier de l'assemblage de textes que constitue le recueil.

Cette palette de poèmes regroupés dans un seul livre est élaborée par le truchement d'une composition spécifique où chacun fournit un élément du sens

pris des choses» à «l'objeu», in Ecritures de l'objet, sous la direction de Roger NAVARRI, Presses Universitaires de Bordeaux, 1997, p. 128.

${ }^{19}$ GENETTE, Gérard, Seuils, éditions du Seuil, coll. Poétique, 1987, p.p. 7,8.

${ }^{20}$ L'emploi du néologisme «titrologie » revient à Claude DUCHET. Plusieurs théoriciens s'intéressent, par la suite, à ce sujet comme Léo HEK, Charles GRIVEL et Roland BARTHES. Cf. respectivement : DUCHET, Claude, La Fille abandonnée et La Bête humaine, éléments de titrologie romanesque, in Littérature, numéro 12, 1973, p.p. 49-73 ; HEK, Leo, La marque du titre, New York, Mouton, 1981, p. 17 et GRIVEL, Charles, Production de l'intérêt romanesque, Mouton, 1973, p. 143 et BARTHES, Roland, Analyse textuelle d'un conte d'Edgar Poe, in Sémiotique narrative et textuelle, Larousse, 1973, p. 34.

${ }^{21} C f$., BARTHES, Roland, op. cit., loc. cit. 
global de l'œuvre tout en se posant comme «noyau unitaire $»^{22}$. Dans la poétique du recueil, il est de coutume d'emprunter le titre de l'un des textes afin de l'appliquer à l'ensemble. Or, Le parti pris des choses échappe à cette tradition puisque l'auteur choisit un titre collectif pour qualifier une association de poèmes soigneusement concertée par l'écrivain. Ainsi, ce titre général de l'œuvre doit refléter à la fois l'unicité et la cohésion de la multitude de poèmes malgré la diversité qui en distingue chacun.

Selon le dictionnaire Le Littré, le terme « parti » est étymologiquement dérivé de l'italien «partito » qui veut dire une partie. Le participe passé du verbe partir, qui signifiait diviser ou partager en morceaux, a historiquement évolué et s'est transformé en substantif « le parti » suggérant ainsi le lot, la part ou la résolution. Alors, prendre parti laisse entendre une prise de position ou une adhésion subjective à un point de vue particulier ${ }^{23}$. La même idée insinue également le concept de défense et de protection, de préférence ou d'appui. Cette structure mystérieuse peut être comprise de deux manières différentes : soit une personne inconnue s'unira à ces éléments et entreprendra la mission de les défendre ; soit que ces «choses », objets sans conscience, prendront ellesmêmes parti et agiront ${ }^{24}$. Un tel titre énigmatique et imprécis invite le lecteur à se demander au sujet de cette pluralité de textes et l'incite à entamer la lecture du recueil.

Le second mot-clé du titre est le terme « chose », mot générique, globale et indéfini. De quel type de choses s'agit-il donc ? Cette impression de vague créée avant la lecture n'est que provisoire, elle demeure en attente d'être clarifiée. En effet, les sujets des poèmes sont des objets très variés entre plantes, êtres vivants et non vivants, personnes, éléments naturels, etc.

Pour examiner les sujets dont il s'agit, nous nous réfèrerons aux titres intérieurs et nous nous appuierons ainsi sur la table des matières. Selon GENETTE, cette dernière n'est « rien d'autre qu'un instrument de rappel de

${ }^{22}$ LANGLET, Irène, Le recueil comme condition ou déclaration, de littéralité : Paul Valéry et Robert Musil, in Etudes littéraires, volume 30, numéro 2, 1998, p. 26.

${ }^{23}$ Cf., dictionnaire Hachette encyclopédique, 2001, p. 1395.

${ }^{24}$ Effectivement, l'auteur adhèrera à la première proposition et son objectif sera "le souci de la chose et le parti-pris d'en faire l'objet essentiel de la pratique poétique » comme nous verrons au cours de notre analyse. ROMESTAING, Alain, art. cit., p. 129. 
l'appareil titulaire $»^{25}$, néanmoins, elle permet une interprétation globale du contenu à travers la vue d'ensemble qu'elle assure. Non seulement elle facilite le repérage de certains détails mais aussi elle offre une opportunité supplémentaire d'analyse et d'interprétation.

En nous basant sur les intertitres, nous avons examiné la structure titrologique générale que nous avons trouvée conforme à l'usage fréquent qui favorise les structures nominales. Sur trente-deux poèmes, "Les arbres se défont à l'intérieur d'une sphère de brouillard» est l'unique titre verbal, il est aussi notoirement le plus long du recueil. Quant à la construction syntaxique, la majorité obéit à la structure la plus simple et la plus commune d'un syntagme nominal construit à partir d'un article défini suivi d'un substantif selon la formule (déterminant + nom). Citons à titre d'exemple L'huître, Le pain, Le gymnaste et $L a$ crevette. Parfois, à cette structure s'ajoute un adjectif tels $L a$ jeune mère et Les trois boutiques. Le déterminant peut aussi disparaitre comme dans Pauvres pêcheurs. La même composition est parfois étalée par un complément de nom, c'est le cas de La fin de l'automne, Les plaisirs de la porte ou Le morceau de viande. Dans quelques textes, nous remarquons l'omission de l'article et la réduction du titre à un seul nom commun comme Pluie, Végétation, Escargots, ce qui nous rappelle la disposition des entrées du dictionnaire et dans d'autres, un complément de nom est assorti au substantif sans déterminant, c'est le cas de Bords de mer et Notes pour un coquillage. La dernière catégorie est celle des noms propres qui présentent des lieux familiers à l'auteur ${ }^{26}$ comme R.C. Seine $n^{\circ}$ et Le restaurant Lemeunier rue de la chaussée $d^{\prime}$ Antin. Ainsi, nous avons conclu que les intertitres dont use PONGE sont des syntagmes de désignation simples, brefs et purement nominaux.

Sous un autre angle, en nous basant sur les titres intérieurs, nous avons opté pour l'esquisse d'un schéma de catégorisation de ces objets afin de distinguer et regrouper thématiquement les entrées de la table des matières exposées dans le recueil. Effectivement, nous avons pu distinguer sept catégories indépendantes qui puissent rassembler les sujets des textes. Nous avons schématisé cette tentative de classification par le truchement du tableau suivant :

${ }^{25}$ GENETTE, Gérard, op. cit., p. 292.

${ }^{26}$ PONGE fréquentait ces lieux lors de son emploi aux Messageries Hachette. 
Rhétorique du monde muet dans « Le parti pris des choses » de Francis PONGE

\begin{tabular}{|c|c|c|c|c|c|c|}
\hline $\begin{array}{c}\text { Les éléments/ } \\
\text { phénomènes de la } \\
\text { nature }\end{array}$ & $\begin{array}{c}\text { Les } \\
\text { personnages }\end{array}$ & Les végétaux & Les objets & $\begin{array}{c}\text { Les } \\
\text { aliments }\end{array}$ & $\begin{array}{l}\text { Les êtres non- } \\
\text { humains }\end{array}$ & Les lieux \\
\hline 1-Pluie & $\begin{array}{l}\text { 3-Pauvres } \\
\text { pêcheurs }\end{array}$ & $\begin{array}{l}\text { 4-Rhum des } \\
\text { fougères }\end{array}$ & 6-Le cageot & 13-Le pain & 10-L'huître & 25-R.C. Seine $n^{\circ}$ \\
\hline $\begin{array}{l}\text { 2-La fin de } \\
\text { l'automne }\end{array}$ & $\begin{array}{l}\text { 23-Le } \\
\text { gymnaste }\end{array}$ & 5-Les mûres & 7-La bougie & $\begin{array}{l}22-\mathrm{Le} \\
\text { morceau } \\
\text { de viande }\end{array}$ & $\begin{array}{l}\text { 16-Le } \\
\text { mollusque }\end{array}$ & $\begin{array}{l}\text { 26-Le restaurant } \\
\text { Lemeunier rue } \\
\text { de la chaussée } \\
\text { d'Antin }\end{array}$ \\
\hline $\begin{array}{l}\text { 12-Les arbres se } \\
\text { défont à l'intérieur } \\
\text { d'une sphère de } \\
\text { brouillard }\end{array}$ & $\begin{array}{l}\text { 24-La jeune } \\
\text { mère }\end{array}$ & 9-L'orange & 8-La cigarette & & 17-Escargots & $\begin{array}{l}\text { 28-Les trois } \\
\text { boutiques }\end{array}$ \\
\hline 14-Le feu & & 19-La mousse & $\begin{array}{l}\text { 11-Les plaisirs } \\
\text { de la porte }\end{array}$ & & 18-Le papillon & \\
\hline $\begin{array}{l}\text { 15-Le cycle des } \\
\text { saigons }\end{array}$ & & 31-Végétation & & & $\begin{array}{l}\text { 27-Notes pour } \\
\text { un coguillage }\end{array}$ & \\
\hline 20-Bords de mer & & & & & 30-La crevette & \\
\hline \multicolumn{7}{|l|}{ 21-De l'eau } \\
\hline \multicolumn{7}{|l|}{ 29-Faune et flore } \\
\hline \multicolumn{7}{|l|}{ 32-Le galet } \\
\hline 9 poèmes & 3 poèmes & 4 poèmes & 4 poèmes & 3 poèmes & 6 poèmes & 3 poèmes \\
\hline
\end{tabular}

Typologie des titres/objets dans Le parti pris des choses

La taxinomie précitée rend parfaitement identifiables les récurrences thématiques. Toutefois, nous pouvons aisément repérer des similitudes dans les catégories. Ainsi, l'inscription dans l'une n'exclut pas les autres puisqu'un même poème peut appartenir simultanément à deux classes différentes. Citons à titre d'exemple L'orange qui pourrait se classifier parmi les végétaux ou les aliments. C'est également le cas du galet qui pourrait se placer avec les objets ou les éléments naturels. En effet, nous les avons répertoriés dans les groupes qui nous ont parus les plus appropriés.

Les objets des textes se rapportent à des classes diverses comme les éléments ou les phénomènes naturels, les personnages, les plantes ou les végétaux, les objets, les aliments ou les produits de consommation, les êtres vivants non-humains et enfin les lieux. Aussi, avons-nous numéroté les poèmes d'après leur ordre d'apparition dans le recueil afin de faciliter le repérage des résurgences des mêmes thèmes ; opération qui semble difficile lors d'une lecture linéaire conforme à la disposition ordinaire des poèmes dans l'ouvrage. Or, nous n'avons pu constater aucune hiérarchisation dans la succession des sujets, leur ordre n'assure point de classement. Ces éléments nous ont parus éparpillés de manière aléatoire sans aucun enchaînement identifiable ${ }^{27}$ mais la multiplicité évidente des objets traités témoigne de la diversité des sources d'inspiration. Sur le plan formel, en examinant la pagination des poèmes nous avons remarqué

${ }^{27}$ C'est à Jean PAULHAN, éditeur et ami de PONGE, que revient le mérite d'avoir assemblé et organisé ces poèmes que l'auteur avait mis 15 ans à composer. 
qu'ils s'allongent vers la fin du recueil. Les textes qui ouvrent l'ensemble sont concis formés de quelques lignes ou même d'un seul paragraphe alors que ceux qui le closent sont visiblement plus longs. Le galet qui est le dernier poème s'étale sur onze pages.

Néanmoins, en dépit du désordre apparent des textes, une réflexion fouillée nous révèle quelques remarques pertinentes. En observant le tableau de catégorisation thématique, nous distinguons une nette préférence pour les sujets faisant partie de la nature ${ }^{28}$ auxquels PONGE consacre neuf poèmes. À cette classe s'ajoute également celle des végétaux et des êtres non-humains ce qui augmente la proportion destinée aux éléments naturels. Les quatre éléments de Bachelard (l'eau, l'air, le feu et la terre) sont dispersés dans le recueil ainsi que les règnes animal et végétal ${ }^{29}$ avec lesquels ils entrent en résonance. En d'autres termes, le champ sémantique autour duquel gravite la majorité des titres est celui de la nature avec quelques exceptions qui sont de véritables choses, inertes fabriquées par l'homme à l'exemple du pain, de la cigarette ou de la bougie. Ainsi donc, l'auteur amalgame subtilement des « choses » hétéroclites à travers un jeu d'échos que se revoient les poèmes tissant une sorte de liens hypertextuels qui reflètent les répercussions structurelles et thématiques.

Dans l'espace qui suit, nous entreprendrons de repérer le système analogique qui étaye le parallélisme entre l'objet et les expressions qui le présentent. Pour ce faire, nous avons choisi des textes appartenant chacun à une catégorie différente ${ }^{30}$. Le premier critère de sélection est que nous avons voulu aborder des poèmes qui n'ont pas été usés par les critiques mais qui, en même temps, se sont révélés riches à l'analyse. D'emblée, nous avons tenté de choisir des textes de longueurs différentes entre succincts et condensés d'une part ou

${ }^{28}$ Une thèse de doctorat a été entièrement consacrée à ce thème où l'emploi de la majuscule à l'initiale du mot Nature souligne d'emblée l'importance. $C f$., CHONWOO, Lee, La Nature dans l'œuvre de Francis Ponge, Université de la Sorbonne nouvelle Paris III, 2011.

${ }^{29} C f$., BALLESTRA-PUECH, Sylvie, "Fragment de la nature des choses" et" inachèvement perpétuel": l'écriture du monde selon Francis Ponge ", in Loxias, numéro 41, mis en ligne le 15 juin 2013, consultable à travers le lien http://revel.unice.fr/loxias/index.html?id=7471, consulté le 21 mai 2020, p. 6.

${ }^{30}$ Afin d'éviter toute redondance inutile et pour ne pas trop étendre le corpus, seuls 7 poèmes retiendront notre attention. Par souci de précision et dans le but de souligner leur disparité, nous les avons sélectionnés dans des classes différentes et nous les avons marqués en caractère gras sur le tableau de catégorisation. Cf., Supra., p. 10. 
longs et développés de l'autre. Ceci afin de pouvoir éclairer les analogies qui mettent en accord cette diversité. Ainsi, nous avons trié un ensemble d'objets poétiques que voici : Les mûres (p. 17, 149 mots), Les plaisirs de la porte (p. 24, 116 mots), Le papillon (p. 40, 149 mots), Bords de mer (p. 41, 680 mots), Le morceau de viande (p. 47, 79 mots), Le gymnaste (p. 48, 127 mots) et Les trois boutiques (p. 65, 290 mots).

\section{Du texte à l'objet :}

«Ponge a rompu avec la psychologie, les sentiments, le destin, l'amour, la mort, la métaphysique, et toutes les grandes choses. Pour pouvoir faire plus grand, il s'est abaissé jusqu'aux petites : les objets, ou les choses $»^{31}$.

Comme nous l'avons déjà signalé ${ }^{32}$, l'auteur avait l'intention de composer des «descriptions-définitions esthétiquement et rhétoriquement adéquates $»^{33}$, nous nous appuierons donc sur cette affirmation dans la partie suivante afin de souligner la manière à travers laquelle l'auteur commence à définir l'objet qui est en même temps le sujet du poème. Dans ce but, nous analyserons respectivement deux stratégies pongiennes différentes : l'observation et la description, car le regard contemplatif, tout comme le langage, pourrait être une sorte de médiation pour exprimer le monde extérieur selon différents points de vue $^{34}$.

PONGE incarne «la survivance de la poésie scientifique $»^{35}$, bien que les objets qu'il traite ne soient pas de nature scientifique, mais le cheminement sur

${ }^{31}$ DRILLON, Jacques, "La pierre Ponge », Entretien à l'occasion de la publication du deuxième volume des Euvres complètes de Francis Ponge dans la Pléiade, in Le Nouvel Observateur, 5 décembre 2002, version électronique disponible à travers le lien http://www.pileface.com/sollers/imprime.php3?id_article=432, consulté le 3 mai 2020.

${ }^{32}$ Cf., Supra, p. 5.

${ }^{33}$ PONGE, Francis, Méthodes, Gallimard, Idées, 1971, p. 20.

${ }^{34}$ N'oublions pas que la rédaction de ces poèmes est imprégnée par le remarquable mouvement de progrès scientifique au XXème siècle. L'effet de la science à long terme sur la société y est présenté à travers une méthodologie déductive qui jette son dévolu sur la logique interne du développement des idées. L'optimisme et la foi de l'érudition et du savoir influencent ainsi les productions littéraires.

${ }^{35}$ RIVA, Magali, art. cit., p. 441. 
lequel il se base rappelle celui des sciences exactes ${ }^{36}$ auquel il emprunte la méthode et la rigueur. Ce genre «poético-scientifique $»^{37}$ est à l'origine de la poésie impersonnelle prônée par l'auteur s'opposant à ce qu'il qualifie de " lyrisme mou ${ }^{38}$. La première étape dans la poétique pongienne est celle de l'observation. Cette activité de contemplation active permet à l'écrivain de reformuler les explications des éléments qu'il présente.

Dans Bords de mer, le texte commence par une phrase que voici : « $L a$ mer jusqu'à l'approche de ses limites est une chose simple qui se répète flot par flot » (Bords de mer, 41). C'est ainsi que se définit le terme «mer» qui est selon le Littré une vaste étendue d'eau salée qui baigne toutes les parties de la terre ${ }^{39}$. Cette ouverture bien que très simpliste retranscrit une idée que nous pourrions voir comme une image. Elle incarne par les lettres le mouvement des vagues qui se précipitent sur la plage par une accumulation de termes plus ou moins de même sens. L'expression «flot par flot » signale une action itérative et scrute visiblement le flux et le reflux de l'eau de la mer. L'écrivain poursuit sa tentative à travers une seconde explication : "Parfois à la rencontre d'un muscle énergique une lame ressort peu à peu : c'est ce qu'on appelle une plage » (Bords de mer, 41), une définition qui vient appuyer la première. C'est le battement puissant et dynamique des cours d'eau qui fait naître les ondes. L'expression « реи à peu » donne une impression de succession qui va de pair avec le débordement des vagues qui courent sur le sable. C'est de cette manière qu'est formulée l'image générale de l'objet «bords de mer ». Ainsi, l'auteur présente des explications dès le début du poème et les multiplie différemment à plusieurs reprises. Plus loin, il va jusqu'à l'analyse de la nomination de cet élément : "C'est en effet, après l'anarchie des fleuves, à leur relâchement dans le profond et copieusement habité lieu commun de la matière liquide, que l'on a donné le nom de mer». Dans cette citation, nous pouvons constater un certain sens latent. Le terme « anarchie » renvoie à un esprit envahi par le désordre et la

${ }^{36}$ Contrairement aux poètes scientifiques, PONGE ne traite pas la science comme sujet de ses poèmes mais il applique la méthodologie scientifique dans le traitement non seulement de l'objet du poème mais bien au-delà, il use d'une technique similaire dans l'analyse de la matière même du poème à savoir le langage comme nous le montrerons dans le troisième volet de notre article. $C f$., infra, p. 17.

${ }^{37}$ RIVA, Magali, art. cit., p. 442.

${ }^{38}$ SOLLERS, Philippe, op. cit., p. 58.

${ }^{39} C f$., Dictionnaire Le Littré en ligne disponible à travers le lien www.littre.org, consulté le 27 avril 2020. 
confusion, or, celui de la «mer », employé au sens figuré, fait allusion à l'immensité de l'érudition, de la connaissance et de la culture, lesquels sont liés, à leur tour, au savoir scientifique. La même idée est renforcée par l'emploi du terme «matière liquide », l'un des trois états chimiques de la matière. C'est ainsi que l'auteur compose des définitions scientifiques illustrées à travers un style purement littéraire.

Le texte du Morceau de viande s'ouvre également par une définition : « Chaque morceau de viande est une sorte d'usine, moulins et pressoirs à sang. Tubulures, hauts fourneaux, cuves y voisinent avec les marteaux-pilons, les coussins de graisse ». (Le morceau de viande, 47) Notons que l'entrée du récit n'est point poétique, elle abonde en vocabulaire scientifique spécialisé et énumère plusieurs machines et outils, on dirait une usine réelle ${ }^{40}$ : moulins, pressoirs, tubulures, fourneaux, marteaux-pilons. L'observation du processus de cuisson de la viande se poursuit à travers le poème et aboutit dans la toute dernière phrase à des "réactions chimiques [qui] se produisent, [et] qui dégagent des odeurs pestilentielles », comme s'il s'agissait d'une expérience dans un laboratoire. Cette impression antipoétique réfléchit bien le titre du poème puisqu'il n'est pas habituel qu'un objet littéraire soit désigné par un titre pareil.

Dans Le papillon, la phrase liminaire est une véritable image de la métamorphose et de la transformation en un être vivant : "Lorsque le sucre élaboré dans les tiges surgit au fond des fleurs (...) un grand effort se produit par terre d'où les papillons tout à coup prennent leur envol ». (Le papillon, 40) Ce début de texte expose une observation des étapes de la chenille qui se transforme en chrysalide s'abritant dans son cocon puis se libère enfin et commence à voler. L'explication de ces différents états se fait progressivement à travers l'usage d'une série de connecteurs qui assurent cette démarche illustrative : lorsque, d'où, mais, dès lors, et d'ailleurs, ainsi. En plus, l'objet du poème n'est nommé qu'à la fin de la première phrase pour créer un certain effet

${ }^{40}$ Il s'agit là d'une « usine biologique » qui connote le cycle de la dégradation de la vie jusqu'à la mort. La même idée sera élucidée dans le volet suivant. $C f$., ADAM, JeanMichel, "Ponge rhétoriquement », in Ponge résolument, actes de colloque sous la direction de J.-M. GLEIZE, Lyon, éditions ENS, 2004, p.p. 19-38. Une version électronique de l'article est disponible à travers le lien https://www.unil.ch/files/live/sites/fra/files/shared/PONGE_Adam.pdf, consulté le 19 mai 2020 , p. 9. 
d'attente. Sur un autre plan, le papillon est un objet littéraire par excellence, il incarne le miracle de la renaissance et de la vie après la mort. C'est ainsi que s'entremêlent l'écriture poétique et l'observation qui est une stratégie purement scientifique.

Quant aux Plaisirs de la porte, le poème commence par une phrase courte qui peut passer pour une vérité générale, «Les rois ne touchent pas aux portes » (Les plaisirs de la porte, 24), elle met en évidence la banalité de l'élément en question et va même jusqu'à déprécier sa valeur. Les rois n'entreprennent jamais l'activité d'ouvrir et de fermer les portes, ils laissent ce travail désagréable à la conciergerie, aux serviteurs et aux officiers de garde. Or, ils se privent ainsi du bonheur de «tenir entre [1]es bras une porte » (Les plaisirs de la porte, 24) puisque le contact quotidien avec cet objet donne une impression de sécurité et fait naître des sentiments rassurants.

Enfin, dans Les trois boutiques, l'exemple de la méditation du bois illustre le trajet de sa composition. Il est «brun, parce que le brun est entre le vert et le noir sur le chemin de la carbonisation »(Les trois boutiques, 65). Cette contemplation expose les étapes de fabrication du bois et en définit chacune par l'association à une couleur dans le processus de fabrication. Ainsi, chez PONGE, «l'écriture doit se travailler sans cesse, certes afin de mieux coller aux choses $»^{41}$.

Tous les exemples mentionnés soulignent que l'auteur fait appel au sens de l'observation afin de définir les objets de ses poèmes. "Les choses, par làmême, accèdent à ce que Ponge appelle un état de rigueur, une tenue hors de leur contenant $\gg^{42}$. La deuxième étape est celle de la description méticuleuse, elle contribue de façon essentielle à l'esquisse des définitions des éléments choisis. Simultanément, elle occupe une place de choix dans tout récit poétique. «Nommer les choses par une technique de la description est un moyen de justifier l'univers et, en même temps, la fonction créatrice $»^{43}$. Ainsi, la phase descriptive constitue un point commun entre l'approche scientifique et littéraire.

${ }^{41}$ ROMESTAING, Alain, art. cit., p. 126.

42 JONGEN, René-Marie, Francis Ponge et la question langagière, Presses de l'Université Saint-Louis, Bruxelles, 2002, p. 374.

${ }^{43}$ LEONARD, Albert, Ponge et la naissance d'une nouvelle rhétorique, in Liberté : Poésie, théâtre, nouvelles, volume 15, numéro 5 (89), 1973, p. 150. 
Le « descriptif ${ }^{44}$ permet de saisir les qualités particulières de l'objet. Toutefois sa technique apparaît dans chacun des poèmes sous un aspect extrêmement novateur voire particulier. Le gymnaste dispose d'une ouverture à dominante descriptive par excellence qui occupe presque la moitié du texte : " Ponge procède souvent par définition de mot, par description inductive de l'objet et par interrogation métalangagière de l'une et de l'autre ${ }^{45}$. Toutefois, cette stratégie n'est pas employée dans un sens objectif, bien au contraire, elle abonde en marques de subjectivité :

« Comme son G l'indique le gymnaste porte le bouc et la moustache que rejoint une grosse mèche en accroche-cour sur un front bas. Moulé dans un maillot qui fait deux plis sur l'aine il porte aussi, comme son $Y$, la queue à gauche ». (Le gymnaste, 48$)$

Le portrait de l'acrobate est exprimé par gradation. Tout d'abord, l'aspect physique est soulevé dès la première phrase à travers la description du gymnaste ayant une barbiche, une moustache et une mèche qui tombe sur le front ${ }^{46}$. Ensuite, dans la deuxième phrase, l'écrivain commence à décrire sa tenue ; il porte un maillot qui enveloppe son organe sexuel ${ }^{47}$. Enfin, il s'intéresse aux gestes de l'athlète à travers son portrait en mouvement. Comme toutes les acrobaties se font à l'aide d'outils comme «la corde à nœuds » et «l'agrès »,

44 Philippe HAMON préfère cet adjectif substantivé suggérant un travail sur l'ensemble plus large de fonctions et de procédés à construire, au terme «description » désignant, selon lui, plus étroitement une unité stylistique identifiable, intégrée à un texte plus vaste. $C f$., HAMON, Philippe, Entretien Le descriptif, « ce délaissé de l'impérialisme Narratologique... », propos recueillis par Guillaume BELLON, in Recto/Verso, Revue de jeunes chercheurs en critique génétique, numéro 7, septembre 2001, p. 1.

45 JONGEN, René-Marie, op. cit., p. 376

${ }^{46}$ Précisions que PONGE analyse le rapport entretenu par le mot «gymnaste » et l'acrobate auquel il renvoie à travers le parallélisme entre la lettre initiale du mot et la physionomie du personnage. Vu de profil, le visage du gymnaste ressemble à la lettre G comme si l'acrobate avait la tête baissée vers le sol.

${ }^{47}$ Là aussi, l'auteur dresse un rapprochement entre la « chose » et le moyen d'expression où l'objet est envisagé par le truchement des lettres qui le désignent. PONGE use ainsi de la représentation graphique de la lettre $\mathrm{Y}$ afin de faire allusion au phallus de l'homme. Cette technique fait preuve d'« une double exigence de vérité, vérité face au monde et vérité face au langage. » JONGEN, René-Marie, op. cit., p. 369. 
l'auteur utilise donc une série de verbes d'action pour illustrer ces exercices : il « bondit », il «choit » puis il « rebondit ». En dépit des étapes de progression logique qu'elle suit, cette pratique descriptive est remarquablement dépréciative car elle présente une image dévalorisante du personnage et en fait l'emblème de la bêtise humaine. Non seulement cela mais elle présente l'acrobate déshumanisé, rabaissé au rang d'un animal qui est tantôt assimilé à un singe tantôt à un ver ou encore à une chenille ${ }^{48}$.

Dans Les mûres, l'auteur utilise toute une palette d'adjectifs chromatiques qui sont des piliers essentiels dans sa description : "Noirs, roses et kakis ensemble sur la grappe ». (Les mûres, 17) L'énumération de ces couleurs obéit à un ordre chronologique particulier. Les adjectifs sont rangés progressivement : à chaque étape de maturation du fruit «correspond son incarnation chromatique $»^{49}$ jusqu'à ce que les mûres deviennent mûres ${ }^{50}$. C'est grâce à un regard analytique se posant sur l'objet que le lecteur parviendra à discerner l'éloquence de sa représentation littéraire.

Dans Les trois boutiques, le descriptif fait appel aux cinq sens afin de représenter, le plus explicitement possible, l'objet dont il est question. À vrai dire, comme l'indique le titre, il ne s'agit pas dans ce poème d'un seul élément mais de trois magasins ayant chacun une spécialisation ${ }^{51}$. Tout un champ lexical des sens parsème le texte. La vue est explicitée à travers les termes : la contemplation, j'observe, à mes yeux; le toucher est exprimé par l'expression action violente ou divisante. Quant au goût, il est désigné par le biais de l'expression fraîchement coupée et l'odorat par les termes un voile de vapeur ou

${ }^{48} C f$., infra., p. 18 (note 57).

${ }^{49}$ CHALMIN, Ronan, La palette d'Hippocrate. Impressions chromatiques sur La mounine de Francis Ponge, in Littérature, numéro 168, décembre 2012, p. 25.

${ }^{50} \mathrm{Ce}$ jeu de mots entre la nomination du fruit et son processus de mûrissement est présenté par l'écrivain dans la dernière phrase du poème : "Sans beaucoup d'autres qualités, -mûres, parfaitement elles sont mûres-comme aussi ce poème est fait. » (Les mûres, 17) Le premier mot faisant allusion au fruit est typographiquement marqué en caractères italiques dans le poème.

${ }^{51}$ Notons la disposition typographique des paragraphes du texte qui va de pair avec les sujets présentés en adoptant un rythme ternaire. À part le premier paragraphe introductif qui énumère les boutiques et la spécialisation de chacun, chaque séquence du poème présente l'un de ces magasins sans aucun lien logique qui puisse les lier ensemble à part leur localisation «près de la place Maubert». (Les trois boutiques, 65) 
de fumée. Finalement et plus discrètement, l'ouïe est connotée par le truchement des verbes partager et proposer qui sous-tendent une certaine oralité. L'usage de cette stratégie descriptive renforce une pratique scripturale qui se veut très méthodique.

Reste à noter que chez PONGE prédomine un certain désir de transmettre tous les détails se rapportant à la « chose » dont il s'agit. Outre le recours aux sens, nous avons repéré un usage notamment excessif des comparatifs : plus rose que nature, la plus grande discrétion, les choses les plus épaisses, l'autre élément plus stable, un peu plus fort clamée, la qualité la plus secrète, moins adroit qu'un singe, moins fauve et stérile, aussi faciles que sobres et sûres. La liste précitée est tirée de plusieurs poèmes et appuie l'idée de l'exactitude et de l'authenticité de la description puisque l'auteur met en œuvre des rapprochements avec d'autres éléments afin de souligner les caractères bien distincts des objets choisis.

"Ponge efface chaque fois la frontière entre description (le singulier, l'individuel, l'épisodique événementiel) et définition (évocation en profondeur de l'universel de l'objet considéré et ainsi plus défini que décrit) ${ }^{52}$. Après avoir décortiqué la démarche pongienne dans le glissement «descriptions / définitions » à travers l'observation des éléments qui constituent la matière essentielle du poème, nous consacrerons la dernière partie de notre article à la dissection de l'exercice stylistique qui en fait « un objet d'art littéraire » car «le rapport de Ponge à la rhétorique constitue une ligne de lecture de son æuvre aussi importante que les approches philosophiques ou esthétiques $\gg^{53}$.

\section{De l'objet à « son rhétorique »:}

«Ponge se propose de réintroduire le sujet individuel dans la rhétorique qui semble l'exclure. Celle-ci doit être fondée sur le goût et permettre à celui-là de se manifester, de s'affirmer, de s'épanouir ${ }^{54}$.

Dans ce volet, nous tenterons d'examiner les moyens d'expression linguistiques et poétiques qui ont aidé PONGE dans la transmission de la parole des « choses ». Afin d'aborder ce dernier point, nous avons opté pour une

\footnotetext{
${ }^{52}$ ADAM, Jean-Michel, art. cit., p. 17.

53 Ibid., p. 1.

${ }^{54}$ FARASSE, Gérard, VECK, Bernard, op. cit., p. 41.
} 
analyse détaillée des sept poèmes sélectionnés, mais cet espace restreint ne nous permettra pas de tout élucider, raison pour laquelle nous nous contenterons de ne citer qu'un seul exemple pour illustrer chaque figure. Notre objectif essentiel vise à examiner la mesure dans laquelle la rhétorique est mise en œuvre afin de dépeindre la « chose».

Dans la poétique de PONGE, «la rhétorique se définit par la façon dont un auteur s'approprie la langue et s'y loge, elle est ce qu'il y a en nous de plus intime ${ }^{55}$. Sous cette optique, l'écrivain recourt à un usage particulièrement distinct des moyens d'expression littéraires qui constitueront la matière de notre réflexion dans cette section. Ainsi, nous soulignerons la gamme de figures de style créées par l'auteur que nous avons classées par groupes.

De prime abord, les figures par analogie retiendront notre attention. Celles-ci permettent de créer des images séduisantes qui engendrent un certain effet impressionnant car elles rapprochent deux termes associés par une équivalence de sens : "Concernant l'analogie, je dirai que son rôle est important dans la mesure où une nouvelle image annule l'imagerie ancienne ${ }^{56}$, assure PONGE. Nous allons traiter respectivement la comparaison, la métaphore, la personnification et la prosopopée.

La comparaison occupe une place de proue dans les poèmes. Prenons l'exemple suivant, "du chef de son corps pris dans la corde à nouds il interroge l'air comme un ver de sa motte ${ }^{57}$. (Le gymnaste, 48 ) Accroché à sa corde à nœuds et l'escaladant vers le ciel, l'athlète est assimilé à un ver qui grimpe une partie de terre élevée. Cette figure permet de relier deux images éloignées afin de les revêtir une charge remarquablement subjective. Le comparant et le comparé partagent la même technique d'escalade, ce qui aboutit à une image extrêmement innovante. Nous avons déjà évoqué l'idée que

${ }^{55}$ Ibid., p. 42.

${ }^{56}$ PONGE, Francis, Méthodes, op. cit., p. 295.

${ }^{57}$ Cette comparaison pourrait être perçue comme «animalisation » : image rhétorique qui désigne une forme spécifique de comparaison consistant à attribuer à un humain un caractère animal. Le plus fréquemment, elle sert à critiquer. Signalons aussi que les comparaisons envahissent ce poème en particulier : "Comme son G l'indique », " Comme son $Y$ », «Comme un ver » et «Comme une chenille». 
l'acrobate est présenté par un portrait dépréciatif qui le ravale au rang d'un ver de terre et avilit sa valeur ${ }^{58}$ laquelle est appuyée par la comparaison précitée.

De plus, la métaphore constitue un aspect stratégique de la poétique pongienne. Elle présente une image rhétorique sans outil de comparaison tout en permettant de renouveler la conception que nous avons de l'objet. Dans Les mûres, les buissons du fruit accolés ensemble sur la grappe sont comparés à une : «agglomération de sphères »; la tige étant assimilée à une ville et les fruits ronds à une concentration d'habitations composant ses banlieues. Cet usage distingué de la métaphore est en étroite relation avec la disposition du fruit et illustre avec une extrême clarté des formes bien distinctes et nettement articulées.

D'emblée, PONGE manifeste un intérêt particulier pour la personnification qui s'inscrit constamment parmi ses moyens d'expression préférés, voilà pourquoi elle est omniprésente dans tous les poèmes bien qu'il s'agisse d'objets ou de choses. Dans Le papillon, nous trouvons l'exemple suivant : "chaque chenille eut la tête aveuglée et laissée noire, et le torse amaigri ». L'auteur personnifie la chenille et la présente sous les traits d'un être humain ayant une tête et un torse. Ce dernier signifie le buste ou la poitrine d'une personne. En revanche, dans les insectes, il s'agit d'un thorax qui signifie une région intermédiaire du corps ${ }^{59}$ portant les organes locomoteurs qui leur permettent de se déplacer.

La prosopopée est aussi une forme différente de la personnification dans laquelle la parole est donnée à des êtres inanimés. Dans l'exemple suivant : «le flot venu de loin (...) trouve à qui parler. Mais une seule et brève parole est confiée aux cailloux et aux coquillages » (Bords de mer, 42), le flux liquide de la mer confie ses secrets aux coquillages, ainsi cette image fait parler et interagir l'objet personnifié qui est lui-même le sujet du poème à savoir le bord de la mer. Ainsi sont employées les figures par analogie afin de « à la fois rapprocher et séparer, réunir et distinguer $»^{60}$.

En second lieu, viennent les figures de substitution. Elles consistent à remplacer le terme par un autre mot ou une expression lui faisant allusion et qui

${ }^{58} C f$., supra, p. 15.

${ }^{59} C f$., dictionnaire Hachette p. 1867

${ }^{60}$ FARASSE, Gérard, VECK, Bernard, op. cit., p. 48. 
lui est proche d'un point de vue logique. C'est donc la tâche du lecteur de saisir le lien sous-jacent. Cette technique permet à l'auteur d'éviter tout risque de redite sauf les répétitions rhétoriques à des fins spécifiques, car chez PONGE, « l'interdit de la répétition, s'impose comme un diktat ${ }^{61}$. Nous aborderons donc la synecdoque et la périphrase.

La synecdoque est un emploi singulier de la métonymie qui consiste à employer un terme en rapport avec celui que l'auteur veut indiquer comme la partie pour le tout. ${ }^{62}$ Ainsi dans cet exemple : «les oiseaux les apprécient peu, si peu de chose au fond leur reste quand du bec à l'anus ils en sont traversés » (Les mûres, 17), l'expression « $d u$ bec à l'anus » est employée métonymiquement afin de faire allusion au système digestif de l'oiseau. Sous cette optique, l'importance du fruit est présentée par rapport à l'animal non à l'homme qui le consomme naturellement. Même par rapport aux oiseaux, le fruit ne présente que «peu de choses au fond », ne suscitant aucun intérêt particulier. Cette stratégie vise à souligner la modestie du fruit de même que sa banalité.

Dans «Les plaisirs de la porte », l'auteur recourt à un usage original de la périphrase pour désigner la porte à deux reprises. Ce procédé langagier exprime par plusieurs mots un référent qui peut être communiqué par un seul terme tel : «ces grands panneaux familiers » et «ces hauts obstacles d'une pièce ». Son emploi fait appel à la technique cinématographique du gros plan où l'accent est mis sur la hauteur et la grandeur de la porte. La focalisation sur les dimensions de l'objet justifie les sentiments de sécurité engendrés par sa présence qui sont évoqués dans le poème par tout un champ lexical du confort comme "le bonheur d'empoigner », "le corps tout entier s'accommode », « une main amicale », «agréablement l'assure ».

En troisième lieu, nous attaquerons les figures d'amplification et d'insistance qui ont pour rôle d'attirer l'attention sur un trait de caractère unique. La figure d'amplification par excellence est l'hyperbole. Elle consiste à utiliser des procédés d'exagération en employant un terme trop outré pour signaler une chose ou un sentiment, ce caractère peut augmenter ou diminuer excessivement la vérité des choses. Ainsi, dans Le papillon, l'expression «la véritable

${ }^{61} \mathrm{Ibid}, \mathrm{p} .41$.

$62 \mathrm{La}$ synecdoque repose essentiellement sur un rapport d'inclusion. $C f$., FROMILHAGUE, Catherine, Les figures de style, sous la direction de Claude Thomasset, Armand Collin, 2010, p. 61. 
explosion » est employée hyperboliquement afin de retranscrire le moment de la sortie de la chenille de son cocon. Or, dans Le morceau de viande, l'auteur présente les étapes de la cuisson de l'aliment jusqu'au bout, jusqu' « à la mort ». L'allusion à la fin de la vie est évoquée par le truchement d'une hyperbole assimilant la viande cuite à une personne morte. La même image est rendue sensible à travers l'expression «odeur pestilentielle » dont la répugnance qualifie usuellement un cadavre. C'est de cette manière que l'hyperbole peut grossir démesurément les faits dans un sens soit mélioratif soit péjoratif afin de frapper l'esprit du lecteur.

En ce qui concerne les figures d'insistance, nous traiterons la gradation, l'accumulation, le parallélisme et le pléonasme. La première est une succession de termes de même nature énumérés par ordre croissant ou décroissant. Ainsi, dans Les mûres, nous trouvons « Noirs, roses et kakis ensemble sur la grappe », c'est une gradation qui procède de manière ascendante comme nous avons déjà souligné $^{63}$. Quant à la deuxième, elle repose essentiellement sur une juxtaposition de termes présentés dans la disposition d'une série comme : " j'observe les comportements différents à mes yeux du métal, de la pierre précieuse, du charbon, de la bûche, du morceau de viande » (Les trois boutiques, 65). L'ordre de ces éléments équivaut à celui des trois boutiques : Bijouterie, Bois et Charbons, Boucherie ; il crée une impression d'aménagement et met en valeur les «choses » qui seront présentées dans le poème. Pour le parallélisme, il consiste à reprendre une même construction syntaxique dans deux groupes de mots comme dans l'exemple : "mettre beaucoup de formes, faire beaucoup de façons » (Bords de mer, p. 41). Ajoutons aussi la redondance ou le pléonasme qui recourt à deux formulations différentes d'un terme, exprimant doublement la même idée comme dans l'exemple : «La mer (...) est une chose simple qui se répète flot par flot. » (Bords de mer, 41). Cet effet de reprise cherche à appuyer l'image par une force rhétorique supplémentaire. Toutes ces images insistantes reposent essentiellement sur la répétition comme concept de base afin de mettre en relief une idée pressante.

${ }^{63} C f$., supra., p. 16. 
En quatrième lieu, s'imposent les figures d'opposition qui rapprochent deux idées contradictoires, l'antithèse ${ }^{64}$ en est la structure la plus courante car elle associe deux éléments antinomiques. L'exemple suivant l'illustre parfaitement : "pousser devant soi avec douceur ou rudesse l'un de ces grands panneaux familiers, se retourner vers lui pour le remettre en place » ( Les plaisirs de la porte, 24 ). Cette phrase rassemble plusieurs mots antithétiques : douceur et rudesse, pousser devant soi et remettre en place. Elle reflète également l'expression anglaise Home sweet home avec toutes les sensations de commodité, de confort et de sécurité que l'on sent une fois de retour chez soi. De plus, cette opposition pourrait être perçue comme incarnation du mouvement d'oscillation des battants de la porte, elle est aussi renforcée par un rythme binaire $^{65}$ qui étaye la même idée de l'organisation du passage dedans/dehors ${ }^{66}$.

En dernier lieu, nous arrivons aux figures de style qui s'appuient sur des jeux des sonorités intitulées "figures de continuité phonique ${ }^{67}$. Nous remarquons chez PONGE une adéquation des « matérialités verbales ${ }^{68}$ exprimant l'objet sur le plan sonore aussi bien que sur le plan graphique. Citons à titre d'exemple l'homéotéleute qui est une figure de style consistant à employer plusieurs termes qui closent par un même accent résonnant dans un « soutien des finales vocaliques ${ }^{69}$. Ainsi, dans Le gymnaste, l'auteur utilise plusieurs mots qui se terminent par le son «aste»: «Tous les cours il dévaste mais se doit d'être chaste et son juron est baste!». Cette redondance des tonalités rappelle incessamment celle du titre du poème Le gymnaste. Quant aux

${ }^{64}$ L'antithèse compte parmi les figures dites « ambivalentes » car elle repose sur une juxtaposition de mots dont les signifiés sont peu compatibles ou opposés. $C f$., FROMILHAGUE, Catherine, op. cit., p. 52.

${ }^{65}$ Le rythme du texte est une variante stylistique non moins importante que le réseau rhétorique. "A partir de Benveniste, le rythme peut ne plus être une sous-catégorie de la forme. (...). Et comme le discours n'est pas séparable de son sens, le rythme n'est pas séparable du sens de ce discours. Le rythme est organisation du sens dans le discours. » MESCHONNIC, Henri, Critique du rythme, Lagrasse, Editions Verdier, 1982, p. 70.

${ }^{66}$ Nous remarquons dans cette citation un emploi exceptionnel de la consonne sifflante /S/ dans une allitération qui évoque le grincement de la porte : "pousser

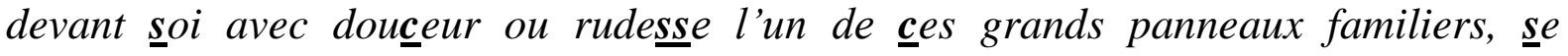
retourner vers lui pour le remettre en place » (Les plaisirs de la porte, 24).

${ }^{67}$ FROMILHAGUE, Catherine, op. cit., p. 22.

${ }^{68}$ JONGEN, René-Marie, op. cit., p. 377.

${ }^{69}$ FROMILHAGUE, Catherine, op. cit., p. 36. 
assonances et aux allitérations, elles reposent respectivement sur la réitération de phonèmes vocaliques ou consonantiques spécifiques. Dans l'exemple suivant : «Un concert élémentaire, par sa discrétion plus délicieux et sujet à réflexion, est accordé là depuis l'éternité pour personne » une assonance en $/ \mathrm{e} /{ }^{70}$ est soulignée à plusieurs reprises. D'emblée, dans cette phrase "il expire en la proférant; et tous ceux qui le suivent expireront aussi en proférant la pareille, parfois par temps à peine un peu plus fort clamée », l'allitération en /p/ prédomine remarquablement. Ces deux exemples sont relevés de Bords de mer afin de souligner que la réapparition des sonorités met en valeur le son doux produit par le mouvement de l'eau et des vagues qui viennent bruyamment mourir sur la plage et effleure des sensations de paix et de bien-être ${ }^{71}$.

Enfin, nous avons la paronomase qui repose sur un rapprochement de deux termes homonymes ayant la même prononciation. Dans cet exemple, " Sans beaucoup d'autres qualités, -mûres, parfaitement elles sont mûres-comme aussi ce poème est fait » (Les mûres, 17), le premier mot désigne le fruit tandis que le deuxième évoque son caractère. Cet effet d'harmonie phonique où le son et le sens vont de pair est tributaire de ce type de jeux de mots ainsi que de celui de la reprise sonore.

Pour synthétiser les principales images abordées dans ce volet, nous les avons rassemblées dans le tableau suivant :

\begin{tabular}{|c|l|}
\hline \multirow{4}{*}{ Les figures par analogie } & La comparaison \\
\cline { 2 - 2 } & La métaphore \\
\cline { 2 - 2 } & La personnification \\
\cline { 2 - 2 } Les figures de substitution & La prosopopée \\
\hline Les figures d'amplification & La synecdoque \\
\cline { 2 - 2 } & La périphrase \\
\hline \multirow{4}{*}{} & L'hyperbole \\
\hline & La gradation \\
\cline { 2 - 2 } & L'accumulation \\
\cline { 2 - 2 } & Le parallélisme \\
\hline
\end{tabular}

${ }^{70}$ Nous employons le symbole phonétique du son.

${ }^{71}$ Cet univers de sensibilité nous rappelle le vers de Baudelaire : «Les parfums, les couleurs et les sons se répondent. » BEAUDELAIRE, Charles, Les fleurs du mal, 1857 (édition princeps), une version numérique de l'œuvre est disponible à travers le lien https://fleursdumal.org/poem/103, consulté le 25 mai 2020. 


\begin{tabular}{|l|l|}
\hline Les figures d'insistance & Le pléonasme \\
\hline Les figures d'opposition & L'antithèse \\
\hline \multirow{4}{*}{ Les jeux sonores } & L'homéotéleute \\
\cline { 2 - 2 } & L'assonance \\
\cline { 2 - 2 } & L'allitération \\
\cline { 2 - 2 } & La paronomase \\
\hline
\end{tabular}

Par le biais du repérage et de l'analyse de cette panoplie de figures de style très diversifiées, nous pouvons confirmer que l'auteur prend plaisir à s'écarter de l'usage ordinaire de la langue. Il investit les moyens d'expression rhétoriques afin de convaincre son lecteur et le séduire par un jeu sur le sens des mots, les constructions phrastiques et les résonances sonores ${ }^{72}$. "Ponge dérègle la rhétorique officielle d'une autre façon en lui faisant subir, outre la contrainte $d u$ goût, celle de l'objet $\gg^{73}$. Les éléments sont révélés, vus et décrits par un langage qui les évoque où s'entrecroisent le fond et la forme. De ce fait, " les objets textuels acquièrent une existence égale à celle des objets naturels $\gg^{74}$. En se faisant le porte-parole d'une nouvelle « chose » dans chaque poème, l'auteur se réjouit à innover et à produire des textes qui se font les échos des objets en question, "cette rhétorique de l'éloge et du blâme n'est que la face superficielle d'un genre d'apparat $\gg^{75}$. La dimension ludique dans la création littéraire est le secret de la poétique pongienne où «l'effort de réflexion critique, solidaire de l'acte d'écriture, est à chaque fois à reprendre $»^{76}$.

À travers ce périple dans Le parti pris de la chose, il est bien évident qu'au-delà de la primauté apparente de l'objet se dissimule la prééminence du langage poétique. L'ambition de l'auteur n'étant donc pas de reproduire des éléments quotidiens mais de fabriquer un nouvel objet poétique en manipulant le texte dans sa matérialité. Dans cette mesure, la « chose » se révèle un simple

${ }^{72}$ PONGE appelle «objeu » cette jubilation du langage qui se déclenche grâce à l'objet. À cette notion se rattache celle de l'« objoie » qui incarne «le pur plaisir du texte ». Cf., COLLOT, Michel, op. cit., respectivement p. 145 - p. 176.

${ }^{73}$ FARASSE, Gérard, VECK, Bernard, op. cit., p. 45.

${ }^{74}$ ROMESTAING, Alain, art. cit., p. 139.

${ }^{75}$ ADAM, Jean-Michel, art. cit., p. 4.

${ }^{76}$ FARASSE, Gérard, VECK, Bernard, op. cit., p. 43. 
prétexte pour se lancer dans une aventure scripturale où la suprématie est celle de l'« objet littéraire ».

Notre étude des principes de cette entreprise pongienne a adopté deux types d'approches sémiotique et stylistique que nous estimons se compléter et s'éclairer mutuellement. Cet angle de vue précis nous a permis d'aboutir à une meilleure explication des « qualités différentielles du texte pongien $»^{77}$ qui sont révélatrices de la tendance artistique de l'écrivain en général. Dans le premier volet «Du titre au texte », nous avons opté pour une étude titrologique des trente-deux textes réunis dans le recueil, laquelle nous a facilité le regroupement des divers éléments présentés par PONGE et nous les avons répertoriés dans des catégories bien autonomes. Par le biais de l'analyse détaillée des titres de ces poèmes, nous avons constaté une parité dans la thématique scripturale qui régit la composition des textes. Nous en avons donc trié sept sur lesquels nous avons focalisé notre attention.

Dans la deuxième partie «Du texte à l'objet », nous avons souligné que l'objet banal qui suscite l'intérêt de l'écrivain demeure essentiel puisqu'il donne son élan à l'écriture. En effet, il est primordial à la technique pongienne que l'œuvre acquiert des qualités objectives. De ce fait, la présentation des caractéristiques particulières et des valeurs inédites de chaque élément a principalement employé deux techniques scientifiques à savoir l'observation et la description. Nous avons conclu que la chose «n'est pas considérée pour ellemême et en elle-même. Elle doit servir désormais à la jubilation de la parole ${ }^{78}$. Ainsi, il nous a fallu voir la mesure dans laquelle le mot et la chose s'entremêlent au point de ne plus pouvoir les extirper.

Compte tenu du fait que la poésie emploie le langage comme moyen d'expression prédominant, dans la dernière section «De l'objet à son rhétorique », nous avons mis en relief que la création littéraire est avant tout une invention de formes d'expressions nouvelles. Ces dernières ont fourni l'objet d'une étude stylistique détaillée des multiples figures de style transposant la beauté singulière des choses, puisque «les plus grands poètes sont ceux qui font voir,

77 JONGEN, René-Marie, op. cit., p. 369.

${ }^{78} C f$., ROMESTAING, Alain, art. cit., p. 136. 
sentir et entendre ${ }^{79}$. Nous avons déduit qu'une même technique scripturale prédomine dans tout le projet du recueil indépendamment du sujet du poème. Durant cet itinéraire, nous sommes partis de la banalité de l'élément qui constitue le sujet du texte pour aboutir à un nouvel objet façonné par les mots.

Francis PONGE exulte de produire des objets-textuels qui « génèrent leurs propres règles de fonctionnement et dont la signification tient à un contexte particulier $\gg^{80}$ et à des usages exceptionnels du langage. Par conséquent, il ne s'agit pas de représenter l'objet mais de constituer le texte comme objet. C'est ainsi que s'éclaire et se justifie l'équation : «Parti pris des choses $=$ compte tenu des mots $»^{81}$. Ce trajet de passage de l'objet quotidien au produit poétique est une entreprise de fabrication non hasardeuse dans laquelle l'auteur remplace la recherche de la réalité par un «bonheur d'expression $»^{82}$ qui puisse lier le contenu et la forme. Cette caractéristique met en évidence « l'effacement de l'objet comme référent ${ }^{83}$ au profit de l'écriture et étaye la propriété singulière du recueil qui pourrait démultiplier les qualités individuelles de toutes ses composantes ${ }^{84}$. Chaque poème du Parti pris des choses est un fragment d'art littéraire qui voit son unicité affirmée et intégrée corrélativement à un ensemble exceptionnel...

${ }^{79}$ ROLLAND DE RENEVILLE, André, L'Expérience poétique : ou le feu secret du langage, Le Grand souffle, 2004, p. 48. Notons que PONGE reçoit le grand prix de poésie de l'Académie française en 1984.

${ }^{80}$ CHARRON, Philippe, op. cit., p. 6.

${ }^{81}$ PONGE, Francis, «My Creative Method», op. cit., p. 522.

${ }^{82}$ CHARRON, Philippe, op. cit., p. 13.

83 BEUGNOT, Bernard, "L'objet médiateur », in Poétiques de l'objet, Champion, 2001, p. 226.

${ }^{84}$ LANGLET, Irène, art. cit., p. 23. 


\section{Bibliographie sélective :}

Nous rappelons que, sauf indication contraire, la ville d'édition dans notre étude est Paris.

I- Corpus : (édition princeps 1942)

PONGE, Francis, Le parti pris des choses, Gallimard, 1967, 170 pages.

Une version électronique est disponible sur le site https://sites.google.com/site/courslettres1eles20172018/home/lpreuve-orale-dubaccalaurat/les-textes-de-posie-de-cette-anne/oeuvre-complte-le-parti-pris-deschoses-de-francis-ponge, consulté le 3 avril 2020.

\section{II- Ouvrages entièrement consacrés à Francis Ponge :}

- CHARRON, Philippe, Représentation de l'objet chez Ponge : une pratique transparente du sens spécifique, Montréal, 2007.

- COLLET, Michel, Francis Ponge : entre mots et choses, Champ Vallon, 1993. - FARASSE, Gérard, VECK, Bernard, Guide d'un petit voyage dans l'œuvre de Francis Ponge, Presses universitaires du Septentrion, Collection Savoirs Mieux, Villeneuve d'Ascq, 1999.

- JONGEN, René-Marie, Francis Ponge et la question langagière, Presses de l'Université Saint-Louis, Bruxelles, 2002.

- SOLLERS, Phillipe, Entretiens de Francis Ponge avec Philippe Sollers, Gallimard, Seuil, 1970.

\section{III- Ouvrages généraux :}

- FROMILHAGUE, Catherine, Les figures de style, sous la direction de Claude Thomasset, Armand Collin, 2010.

- GENETTE, Gérard, Seuils, éditions du Seuil, coll. Poétique, 1987.

- GRIVEL, Charles, Production de l'intérêt romanesque, Mouton, 1973.

- HEK, Leo, La marque du titre, New York, Mouton, 1981.

- LEJEUNE, Philippe, Le pacte autobiographique, Seuils, 1975.

- MAINGUENEAU, Dominique, Le discours littéraire : Parotopie et scène d'énonciation, Armand Colin, 2004.

- MESCHONNIC, Henri, Critique du rythme, Lagrasse, Editions Verdier, 1982.

- ROLLAND DE RENEVILLE, André, L'Expérience poétique : ou le feu secret du langage, Le Grand souffle, 2004.

\section{IV- Articles entièrement consacrés à Francis Ponge :}


- BALLESTRA-PUECH, Sylvie, "Fragment de la nature des choses" et" inachèvement perpétuel" : l'écriture du monde selon Francis Ponge », in Loxias, numéro 41, mis en ligne le 15 juin 2013, consultable à travers le lien http://revel.unice.fr/loxias/index.html?id=7471, consulté le 21 mai 2020.

- CHALMIN, Ronan, La palette d'Hippocrate. Impressions chromatiques sur La mounine de Francis Ponge, in Littérature, numéro 168, décembre 2012.

- LEONARD, Albert, Ponge et la naissance d'une nouvelle rhétorique, in Liberté : Poésie, théâtre, nouvelles, volume 15, numéro 5 (89), 1973.

- PETERSON, Michel, Francis Ponge au cour de son intimité, in Nuit Blanche, numéro 85, 2000-2001.

- RIVA, Magali, Francis Ponge : la méthode poétique, in La poésie scientifique de la gloire au déclin, Epistémocritique, littérature et savoirs, 2014.

- ROMESTAING, Alain, L'objet chez Francis Ponge: du "Parti-pris des choses» à «l'objeu », in Ecritures de l'objet, sous la direction de Roger NAVARRI, Presses Universitaires de Bordeaux, 1997.

\section{V- Articles généraux :}

- BARTHES, Roland, Analyse textuelle d'un conte d'Edgar Poe, in Sémiotique narrative et textuelle, Larousse, 1973.

- BEUGNOT, Bernard, « L'objet médiateur », in Poétiques de l'objet, Champion, 2001.

- DUCHET, Claude, La Fille abandonnée et La Bête humaine, éléments de titrologie romanesque, in Littérature, numéro 12, 1973.

- LANGLET, Irène, Le recueil comme condition ou déclaration, de littéralité : Paul Valéry et Robert Musil, in Etudes littéraires, volume 30, numéro 2, 1998.

\section{VI- Entretiens :}

- DRILLON, Jacques, «La pierre Ponge », Entretien à l'occasion de la publication du deuxième volume des Euvres complètes de Francis Ponge dans la Pléiade, in Le Nouvel Observateur, 5 décembre 2002, version électronique disponible à travers le lien http://www.pileface.com/sollers/imprime.php3?id article=432, consulté le 3 mai 2020.

- HAMON, Philippe, Entretien Le descriptif, « ce délaissé de l'impérialisme Narratologique... », propos recueillis par Guillaume BELLON, in Recto/Verso, Revue de jeunes chercheurs en critique génétique, numéro 7, septembre 2001. 
- SOLLERS, Philippe, "Ponge en abîme », Entretien à l'occasion de la publication des ouvres de Francis Ponge dans la Pléiade en 1999, version électronique disponible à travers le lien http://www.pileface.com/sollers/spip.php?article432, consulté le 3 mai 2020.

\section{VII- Actes de colloque :}

- ADAM, Jean-Michel, « Ponge rhétoriquement », in Ponge résolument, actes de colloque sous la direction de J.-M. GLEIZE, Lyon, éditions ENS, 2004. Une version électronique de l'article est disponible à travers le lien https://www.unil.ch/files/live/sites/fra/files/shared/PONGE Adam.pdf, consulté le 19 mai 2020.

- RODRIGEZ, Antonio, «La référence « classique » chez les "modernes » : les enjeux d'une association antiromantique en poésie (1910-1960) », in Etudes de Lettres, actes du colloque «Francis Ponge » à l'université de Lausanne, sous la direction de André Wyss, numéro 1, 1999. Une version électronique de l'article est disponible à travers le lien https://www.academia.edu/38145240/La_r\%C3\%A9f\%C3\%A9rence_classique _chez_les_po\%C3\%A8tes_modernes.pdf, consulté le 28 mai 2020.

\section{VIII- Thèse :}

- CHONWOO, Lee, La Nature dans l'œuvre de Francis Ponge, Université de la Sorbonne nouvelle Paris III, 2011.

\section{IX- Dictionnaires :}

- Hachette encyclopédique, 2001.

- Le Littré en ligne disponible à travers le lien www.littre.org, consulté le 27 avril 2020.

\section{$\mathrm{X}$ - Autres ouvrages cités :}

- BEAUDELAIRE, Charles, Les fleurs du mal, 1857 (édition princeps). Une version numérique de l'œuvre est disponible à travers le lien https://fleursdumal.org/poem/103, consulté le 25 mai 2020.

- PONGE, Francis, La rage de l'expression, Gallimard, 1952.

- -----------------, Méthodes, Gallimard, Idées, 1971.

- -------o «My Creative Method », in Euvres complètes, tome I,

Gallimard, Bibliothèque de la Pléiade, 1999. 


\section{References:}

\section{$\underline{\text { I- Corpus: }}$}

PONGE, Francis, The Partisanship of things, Gallimard, 1967 (1942), 170 pages.

URL:https://sites.google.com/site/courslettres1eles20172018/home/lpreuveorale-du-baccalaurat/les-textes-de-posie-de-cette-anne/oeuvre-complte-le-partipris-des-choses-de-francis-ponge, accessed April 2020.

\section{II- Books entirely dedicated to Francis Ponge:}

- CHARRON, Philippe, Representation of the object in Ponge's work: a transparent practice of the specific meaning, Montreal, 2007.

- COLLET, Michel, Francis Ponge: between words and things, Champ Vallon, 1993.

- FARASSE, Gérard, VECK, Bernard, Guide to a little trip in the work of Francis Ponge, Press of the Septentrion, Collection Savoirs Mieux, Villeneuve d'Ascq, 1999.

- JONGEN, René-Marie, Francis Ponge and the language issue, Press of the University Saint-Louis, Bruxelles, 2002.

- SOLLERS, Phillipe, Francis Ponge interviews with Philippe Sollers, Gallimard, Seuil, 1970.

\section{III- General books:}

- FROMILHAGUE, Catherine, The figures of speech, director Claude Thomasset, Armand Collin, 2010.

- GENETTE, Gérard, Thresholds, Seuil, coll. Poétique, 1987.

- GRIVEL, Charles, Production of romance interest, Mouton, 1973.

- HEK, Leo, The mark of the title, New York, Mouton, 1981.

- LEJEUNE, Philippe, The autobiographical pact, Seuils, 1975.

- MAINGUENEAU, Dominique, The literary discourse: Parotopia and scene of enunciation, Armand Colin, 2004.

- MESCHONNIC, Henri, Criticism of the rhythm, Lagrasse, edition Verdier, 1982.

- ROLLAND DE RENEVILLE, André, Poetic experience : or the secret fire of language, Le Grand souffle, 2004. 


\section{IV- Articles entirely dedicated to Francis Ponge:}

- BALLESTRA-PUECH, Sylvie, "Fragment of the nature of things " et " perpetual inactivity " : writing of the world according to Francis Ponge », in Loxias, $\mathrm{n}^{\circ}$ 41, URL http://revel.unice.fr/loxias/index.html?id=7471, accessed May 2020.

- CHALMIN, Ronan, The palette of Hippocrates. Chromatic impressions on Francis Ponge's mounine, in Littérature, $n^{\circ}$ 168, december 2012.

- LEONARD, Albert, Ponge and the birth of a new rhetoric, in Liberté: Poésie, thêâtre, nouvelles, volume 15, n 5 (89), 1973.

- PETERSON, Michel, Francis Ponge at the heart of his intimacy, in Nuit Blanche, $n^{\circ} 85,2000-2001$.

- RIVA, Magali, Francis Ponge: the poetic method, in The scientific poetry of glory to decline, Epistémocritique, littérature et savoirs, 2014.

- ROMESTAING, Alain, The object of Francis Ponge: from «The Partisanship of things » to the "objeu", in Writing of the object, director de Roger NAVARRI, Press of University Bordeaux, 1997.

\section{V- General articles:}

- BARTHES, Roland, Textual analysis of a tale by Edgar Poe, in Narrative and textual semiotics, Larousse, 1973.

- BEUGNOT, Bernard, «The subject matter of mediation », in Poetics of the object, Champion, 2001.

- DUCHET, Claude, The Abandoned Girl \& The Human Beast, elements of romanesque titrology, in Littérature, $\mathrm{n}^{\circ}$ 12, 1973.

- LANGLET, Irène, The collection as a condition or declaration of literality: Paul Valéry \& Robert Musil, in Literary studies, volume 30, n 2, 1998.

\section{VI- Interviews:}

- DRILLON, Jacques, "The Ponge stone », in The Nouvel Observateur, 5 december 2002,

URL http://www.pileface.com/sollers/imprime.php3?id_article=432, accessed May 2020. 
- HAMON, Philippe, The description, «this forsaken of Narratological imperialism...», interview with Guillaume BELLON, in Recto/Verso, $\mathrm{n}^{\circ}$ 7, september 2001.

- SOLLERS, Philippe, «Ponge in the abyss », URL http://www.pileface.com/sollers/spip.php?article432, accessed May 2020.

\section{VII- Conference proceedings :}

- ADAM, Jean-Michel, «Ponge rhetorically», in Ponge resolutely, director J.M. GLEIZE, Lyon, editions ENS, 2004. URL https://www.unil.ch/files/live/sites/fra/files/shared/PONGE_Adam.pdf, accessed May 2020.

- RODRIGEZ, Antonio, «The «classical» reference in the «modern»: the stakes of an anti-Romantic association in poetry (1910-1960) », in Studies of Letters, director André Wyss, Lausanne University, n¹, 1999.

URL :https://www.academia.edu/38145240/La_r\%C3\%A9f\%C3\%A9rence_cla ssique_chez_les_po\%C3\%A8tes_modernes.pdf, accessed May 2020.

\section{VIII- Thesis:}

- CHONWOO, Lee, Nature in the work of Francis Ponge, Sorbonne nouvelle University, Paris III, 2011.

\section{IX- Dictionaries:}

- Hachette Encyclopedic dictionary, 2001.

- Littré online, URL www.littre.org, accessed April 2020.

\section{X- $\underline{\text { Other cited books: }}$}

- BEAUDELAIRE, Charles, The Flowers of Evil, 1857 (first edition). URL https://fleursdumal.org/poem/103, accessed May 2020.

- PONGE, Francis, The rage of expression, Gallimard, 1952.

- -------- Methodes, Gallimard, Idées, 1971.

- --Gallimard, Bibliothèque de la Pléiade, 1999. 


\section{بلاغة العالم الصامت في ديوان "تحيز الأشياء" بقلم فرانسيس بونج

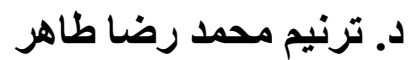 \\ قسم اللغة الفرنسية_كلية التربية-جامعة الإسكندرية}

المستخلص

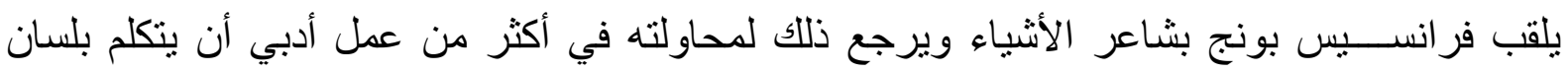

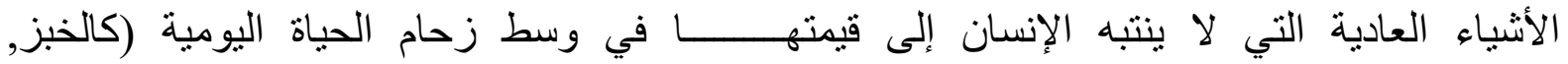

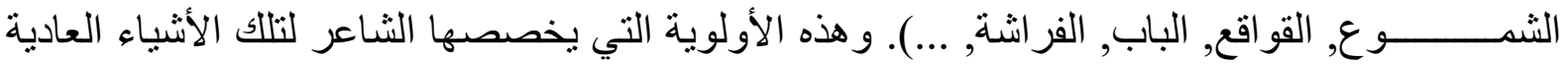

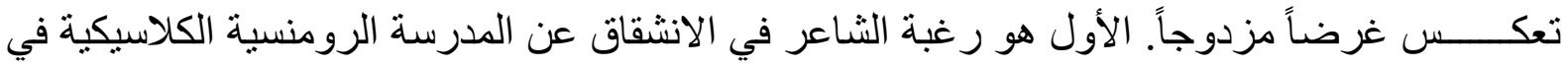

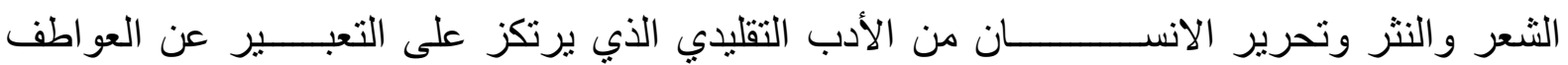

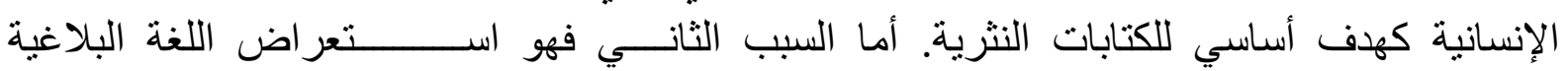

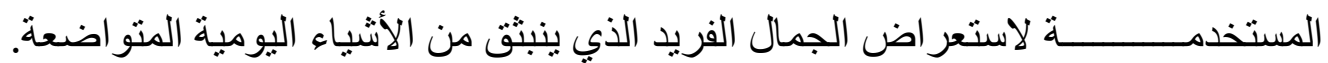

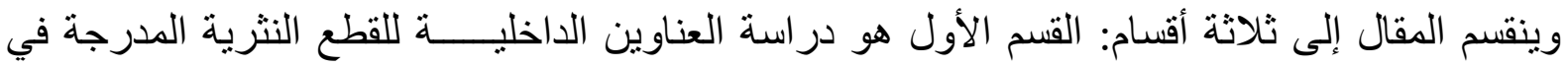

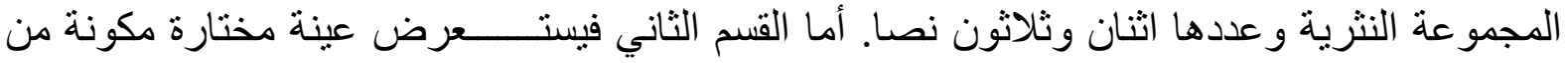

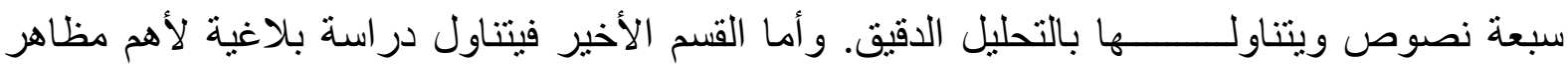

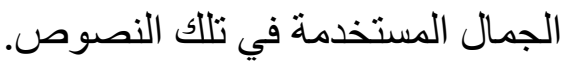

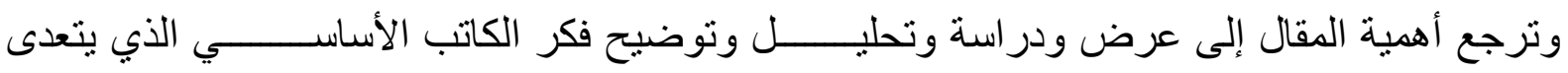

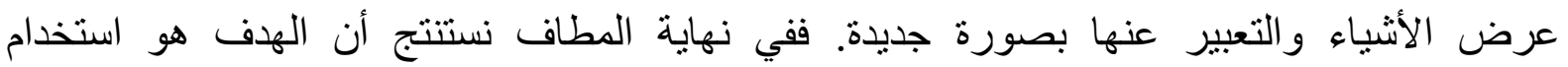

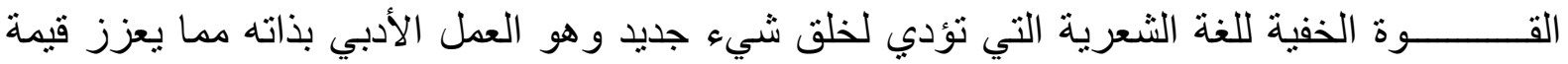

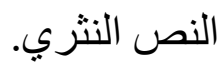

